\title{
Effect of branched-chain amino acid supplementation on the oxidized/reduced state of plasma albumin in rats with chronic liver disease
}

\author{
Masashi Kuwahata, ${ }^{1, *}$ Hiroyo Kubota, ${ }^{1}$ Misaki Katsukawa, ${ }^{1}$ Shunsuke Ito, ${ }^{1}$ Aki Ogawa, ${ }^{1}$ Yukiko Kobayashi, ${ }^{1}$ \\ Yasushi Nakamura² and Yasuhiro Kido' \\ 'Departments of Nutrition Science, Kyoto Prefectural University, 1-5 Shimogamo-hangi-cho, Sakyo, Kyoto 606-8522, Japan \\ ${ }^{2}$ Food Science, Graduate School of Life and Environmental Sciences, Kyoto Prefectural University, 1-5 Shimogamo-hangi-cho, Sakyo, Kyoto 606-8522, Japan
}

\begin{abstract}
We examined whether continuous supplementation with branchedchain amino acids phosphorylates ribosomal protein S6, a downstream effector of mammalian target of rapamycin, and improves hypoalbuminemia of rats with chronic liver disease. SpragueDawley rats were fed a casein diet (control group) or a branchedchain amino acid-supplemented casein diet (branched-chain amino acid group) for 11 weeks with repeated injections of carbon tetrachloride. Throughout this experimental period, no significant difference in plasma albumin concentration was seen between groups. The percentage of reduced albumin within total plasma albumin gradually decreased in both control and branched-chain amino acid groups. After 11 weeks with supplementation, phosphorylation of ribosomal protein S6 was significantly increased in the liver of rats in the branched-chain amino acid group compared with the control group. Furthermore, the percentage of reduced albumin within total albumin was significantly higher in the branched-chain amino acid group than in the control group. These results indicate that continuous supplementation with branchedchain amino acids in rats with chronic liver disease induces phosphorylation of hepatic ribosomal protein $\mathrm{S} 6$ and attenuates decreases in the percentage of reduced albumin, although levels of plasma albumin are not increased.
\end{abstract}

Key Words: branched-chain amino acids, chronic liver disease, reduced albumin, ribosomal protein $\mathrm{S6}$, rats

$\mathrm{P}$ lasma amino acid imbalances, including a decrease in the concentration of branched-chain amino acids (BCAAs), an increase in aromatic amino acids (AAAs), and a subsequent reduction in the molar ratio of BCAAs to AAAs, are characteristic of patients with liver cirrhosis. ${ }^{(1,2)}$ In addition, the reduction of BCAA/AAA ratio correlated strongly with the reduction of plasma proteins, including albumin, transferrin, prealbumin and retinolbinding protein. ${ }^{(3)} \mathrm{A}$ high prevalence of hypoalbuminemia is seen among patients with chronic hepatic failure, including patients with decompensated liver cirrhosis. ${ }^{(4-6)}$ Survival rates are lower in cirrhotic patients with hypoalbuminemia compared with patients showing normal albumin concentrations $(>3.5 \mathrm{~g} / \mathrm{dL}) .{ }^{(7)}$

Albumin displays microheterogeneity, including oxidized and reduced forms of albumin. ${ }^{(8)}$ Human mercaptalbumin (HMA, reduced albumin) has a free thiol form in the cysteine residue at the 34th position from the N-terminal. On the other hand, the cysteine residue in human nonmercaptalbumin (HNA, oxidized albumin) forms a disulfide with small thiol compounds, mainly cysteine or cysteinylglycine (HNA1) or is oxidized with a sulfinic $\left(-\mathrm{SO}_{2} \mathrm{H}\right)$ or sulfonic $\left(-\mathrm{SO}_{3} \mathrm{H}\right)$ acid $(\mathrm{HNA} 2)$. The percentage of HNA within total albumin is about $30 \%$ in serum from healthy subjects. ${ }^{(9)}$ In cirrhotic patients, both albumin synthesis and degradation rates of albumin are decreased, and the biological half-life of albumin is prolonged. ${ }^{(7)}$ As a result, the percentage of HNA within total albumin increases with the progression of liver cirrhosis. ${ }^{(9)}$ In Japan, pharmacological supplementation with BCAAs is widely used to improve hypoalbuminemia in patients with decompensated liver cirrhosis. ${ }^{(10,11)}$ Previous studies have reported that the decreased synthesis and degradation rates and abnormal oxidized/reduced state of albumin can be improved in cirrhotic patients with BCAA supplementation. ${ }^{(7,12)}$

L-leucine, one of the BCAA components, can activate mammalian target of rapamycin (mTOR) signaling, which is critical for protein synthesis at the level of translational initiation. ${ }^{(13,14)}$ Activated mTOR phosphorylates p70 S6 kinase, followed by activated p70 S6 kinase that phosphorylates ribosomal protein S6, and as a result, increases the protein synthesis complex. Activated mTOR also phosphorylates 4E-BP1 and promotes the formation of the protein synthesis initiation complex. Previous studies have suggested that addition of leucine to culture medium promotes albumin synthesis in rat primary hepatocytes ${ }^{(15)}$ and the HepG2 human cell line ${ }^{(16)}$ via the activation of mTOR signaling. Furthermore, oral administration of BCAAs reportedly activates mTOR signaling in the liver of rats with chronic liver disease. ${ }^{(17)}$ However, in that animal experiment, activation of mTOR signaling was evaluated under transient administration of a large amount of BCAAs. This means that evidence is lacking that continuous supplementation with BCAAs as seen in clinical scenarios for cirrhotic patients activates mTOR signaling in the liver of cirrhotic model animals. The present study investigated whether continuous supplementation with BCAAs in rats with chronic liver disease influenced the activation of hepatic mTOR signaling and the oxidized/reduced ratio of plasma albumin.

\section{Materials and Methods}

Animals. The animal facilities and protocol were reviewed and approved by the Institutional Animal Care and Use Committee at Kyoto Prefectural University. Male Sprague-Dawley rats (weight, about $200 \mathrm{~g}$ ) were housed under a 12-h light/dark cycle, with ad libitum access to commercial diet and water. Chronic liver disease was induced by injection of carbon tetrachloride $\left(\mathrm{CCl}_{4}\right)$ (Wako Pure Chem., Osaka, Japan). Briefly, after an acclimatization period of 1 week, $\mathrm{CCl}_{4}$ mixed with an equal volume of olive oil was injected subcutaneously twice a week at a dose of $1.0 \mathrm{~mL} / \mathrm{kg}$

*To whom correspondence should be addressed.

E-mail: kuwahata@kpu.ac.jp 
Table 1. Composition of test diets

\begin{tabular}{|c|c|c|}
\hline Component & Control diet & BCAA diet \\
\hline Casein & 200 & 175 \\
\hline Valine & 0 & 7 \\
\hline Leucine & 0 & 12 \\
\hline Isoleucine & 0 & 6 \\
\hline Cornstarch & 457 & 457 \\
\hline Sucrose & 228 & 228 \\
\hline Rapeseed oil & 35 & 35 \\
\hline Soybean oil & 15 & 15 \\
\hline Cellulose & 20 & 20 \\
\hline Vitamin mixture $^{1}$ & 10 & 10 \\
\hline Mineral mixture $^{2}$ & 35 & 35 \\
\hline
\end{tabular}

${ }^{1}$ AIN-76 vitamin mixture. ${ }^{2} \mathrm{AIN}-76$ mineral mixture.

of body weight for 11 weeks. During this experimental period, rats were divided into 2 groups and fed either the casein diet (control group, $n=5$ ) or the BCAA-supplemented casein diet (BCAA group, $n=6$ ) (Table 1). Rats were given ad libitum access to the diet and drinking water. Dietary intake and body weight were measured every day during the experimental period. Blood was drawn from the tail vein every week and centrifuged to separate plasma. After 11 weeks, each rat was anesthetized with diethyl ether. Blood was drawn and centrifuged to separate plasma. The liver was rapidly removed and weighed. Plasma and liver samples were stored at $-70^{\circ} \mathrm{C}$ until analysis.

Measurement of plasma aminotransferase, total protein and albumin. Plasma concentrations of aspartate aminotransferase, alanine aminotransferase, total protein and albumin were measured using GOT-L, GPT-L, TP-L and BCG-L kits (Cerotec, Sapporo, Japan), respectively. Samples were analyzed using a CL-8000 autoanalyzer (Shimadzu, Kyoto, Japan).

Measurement of plasma amino acids. Plasma was deproteinized with ethanol and the supernatant was used for amino acid analysis by high-performance liquid chromatography (HPLC) using a precolumn phenylisothiocyanate derivatization technique. ${ }^{(18)}$ Norleucine was used as an internal standard. The molar ratio of BCAAs to AAAs was calculated as (Valine + Leucine + Isoleucine) / (Phenylalanine + Tyrosine).

Histological examination. Small pieces of each liver were fixed in $10 \%$ buffered formaldehyde then embedded in paraffin. Paraffin sections ( $3 \mu \mathrm{m}$ thick) were stained with Azan stain to observe liver fibrosis. Sections were imaged using a microscope (Olympus Co., Tokyo, Japan).

Western blot analysis. Western blot analysis using anti-S6 and anti-phospho-S6 antibodies (Ser 235/236) (Cell Signaling Technology, Danvers, MA) was performed as described previously. ${ }^{(16)}$ Briefly, the liver was homogenized in buffer containing $20 \mathrm{mM}$ HEPES (pH 7.4), $100 \mathrm{mM} \mathrm{KCl}, 0.2 \mathrm{mM}$ EDTA, $2 \mathrm{mM}$ EGTA, $1 \mathrm{mM}$ DTT, $50 \mathrm{mM} \mathrm{NaF}, 50 \mathrm{mM} \beta$-glycerophosphate, $0.1 \mathrm{mM}$ PMSF, $1 \mathrm{mM}$ benzamidine and $0.5 \mathrm{mM}$ sodium vanadate. Homogenates were centrifuged at $8,000 \times g$ for $30 \mathrm{~min}$ at $4^{\circ} \mathrm{C}$. The resulting supernatant fraction was subjected to western blot analysis. The protein concentration was determined using BCA protein assay reagent (Pierce, Rockford, IL).

Analysis of the oxidized/reduced state of albumin. HPLC was performed using method described by Hayashi et al. ${ }^{(19)}$ The HPLC system consisted of a \#3023 autosampler (injection volume, $0.5 \mu \mathrm{L}$ ), \#3101 pumps and a \#3213 fluorescence detector (excitation wavelength, $280 \mathrm{~nm}$; emission wavelength, $340 \mathrm{~nm}$ ) in conjunction with a S-MC system controller (all from Shiseido, Tokyo, Japan). An ES-502N ion-exchange column (ShodexAsahipak) (Showa Denko K.K., Kawasaki, Japan) was used.
Measurements were carried out by solvent gradient elution with increasing ethanol concentration from $0 \%$ to $10 \%$ in $50 \mathrm{mM}$ sodium acetate and $400 \mathrm{mM}$ sodium sulfate $(\mathrm{pH} 4.85)$ at a flowrate of $1.0 \mathrm{~mL} / \mathrm{min}$.

Statistical analysis. Data are expressed as mean \pm standard error of the mean (SEM). The uniformity of standard deviation within groups was evaluated using the $F$-test. The significance of differences was assessed using Student's $t$ test. Statistical analysis for multiple comparisons was performed using one-way analysis of variance followed by a Tukey-Kramer post hoc test. Data analysis was performed using Statcel 2 software (Oms Publishing, Tokyo, Japan) and values of $p<0.05$ were considered statistically significant.

\section{Results}

In the experimental period, no significant differences in plasma albumin levels were seen between control and BCAA groups (Fig. 1A). The percentage of reduced albumin gradually decreased from 4 weeks in both control and BCAA groups (Fig. 1B). However, the decrease in reduced albumin was more marked in the control group than in the BCAA group.

No significant differences in food intake, final body weight or liver weight were seen between control and BCAA groups (Table 2). In terms of blood biochemistry, no significant differences in aminotransferase activities, total protein or albumin
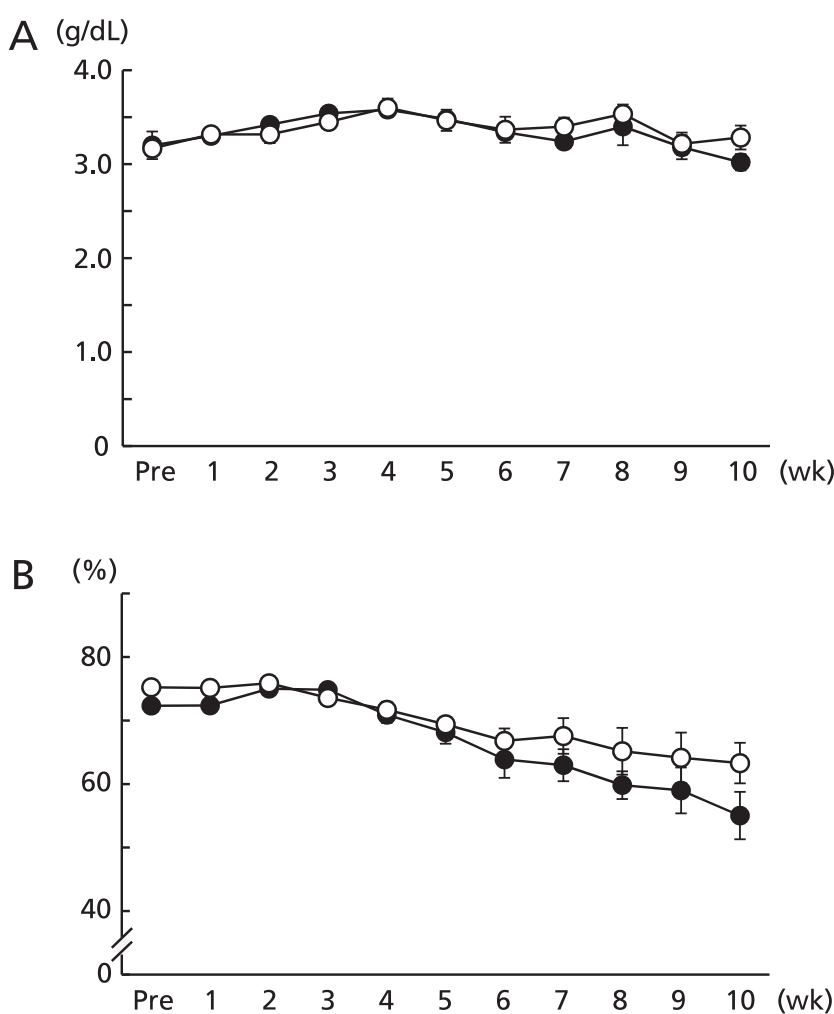

Fig. 1. Effect of BCAA supplementation on levels of plasma albumin and percentages of reduced albumin in $\mathrm{CCl}_{4}$-treated rats. $\mathrm{CCl}_{4}$-treated rats were fed either the control diet (control group, closed circles) or the BCAA-supplemented diet (BCAA group, open circles) for 11 weeks. Blood was drawn from the tail vein every week. Plasma albumin levels were monitored $(A)$ and percentages of reduced albumin within total albumin were analyzed by HPLC (B). HPLC was performed using an ES$502 \mathrm{~N}$ ion-exchange column with an increasing ethanol concentration from $0 \%$ to $10 \%$ in $50 \mathrm{mM}$ sodium acetate and $400 \mathrm{mM}$ sodium sulfate buffer ( $\mathrm{pH} 4.85)$. Values represent means \pm SEM $(n=3)$. 
Table 2. Food intake, body weight, liver weight and blood biochemistry in $\mathrm{CCl}_{4}$-treated rats fed control or BCAA-supplemented diets for 11 weeks

\begin{tabular}{lcc}
\hline & Control & BCAA \\
\hline Food intake (g/day) & $19 \pm 1$ & $21 \pm 1$ \\
Body weight (g) & $365 \pm 18$ & $404 \pm 16$ \\
Liver weight (g) & $15.3 \pm 1.0$ & $19.9 \pm 1.8$ \\
Blood biochemistry & & \\
AST (IU/L) & $916 \pm 93$ & $586 \pm 138$ \\
ALT (IU/L) & $455 \pm 98$ & $522 \pm 208$ \\
Total protein (g/dL) & $5.1 \pm 0.1$ & $5.6 \pm 0.2$ \\
Albumin (g/dL) & $2.7 \pm 0.1$ & $2.9 \pm 0.1$ \\
\hline
\end{tabular}

Values are given as means \pm SEM (Control, $n=5$; BCAA, $n=6$ ). AST, aspartate aminotransferase; ALT, alanine aminotransferase.

Table 3. Plasma BCAA and AAA concentrations in $\mathrm{CCl}_{4}$-treated rats fed control or BCAA-supplemented diets for 11 weeks

\begin{tabular}{lcc} 
& & (nmol/mL) \\
\hline Valine & Control & BCAA \\
Leucine & $208 \pm 34$ & $325 \pm 15^{*}$ \\
Isoleucine & $140 \pm 25$ & $219 \pm 13^{*}$ \\
BCAA & $128 \pm 7$ & $127 \pm 11$ \\
Tyrosine & $476 \pm 62$ & $671 \pm 38^{*}$ \\
Phenylalanine & $126 \pm 21$ & $118 \pm 18$ \\
AAA & $66 \pm 11$ & $68 \pm 9$ \\
BCAA/AAA molar ratio & $192 \pm 32$ & $186 \pm 27$ \\
\hline
\end{tabular}

Values are given as means \pm SEM (Control, $n=3$; BCAA, $n=5$ ). ${ }^{*} p<0.05$.

concentrations were seen between groups (Table 2). $\mathrm{CCl}_{4}$-treated rats developed liver fibrosis (Fig. 2). However, the liver fibrosis was mild in the BCAA group compared with the control group. Plasma valine and leucine levels were significantly higher in the BCAA group than in the control group (Table 3). As a result, plasma BCAA levels were significantly higher in the BCAA group than in the control group (Table 3 ). No significant differences in plasma AAA levels were seen between groups (Table 3 ). The molar ratio of BCAAs to AAAs was significantly higher in the BCAA group than in the control group (Table 3). Phosphorylation of ribosomal protein $\mathrm{S} 6$, a downstream effector of mTOR, was remarkably decreased in $\mathrm{CCl}_{4}$-treated rats compared with normal rats (Fig. 3). However, phosphorylation of ribosomal protein S6 was significantly increased in the BCAA group compared with the control group.

Fig. 4 shows typical HPLC profiles of plasma albumin. In plasma from normal rats, percentages of reduced albumin and oxidized albumin within total albumin were $72.5 \pm 0.8 \%$ and $27.5 \pm 0.8 \%$, respectively $(n=3)$ (Fig. $4 \mathrm{~A})$. In plasma from rats treated with $\mathrm{CCl}_{4}$ for 11 weeks, percentages of reduced albumin and oxidized albumin within total albumin were $51.6 \pm 2.4 \%$ and $48.4 \pm 2.4 \%$, respectively, in the control group (Fig. 4B), and $62.8 \pm 3.0 \%$ and $37.3 \pm 3.0 \%$, respectively, in the BCAA group (Fig. 4C). The percentage of reduced albumin was significantly higher in the BCAA group than in the control group (Fig. 5).

\section{Discussion}

Long-term nutritional supplementation with oral BCAAs improves hypoalbuminemia in patients with liver cirrhosis. ${ }^{(11)}$ The mechanism by which BCAAs improve hypoalbuminemia has been thought to involve promotion of albumin synthesis via
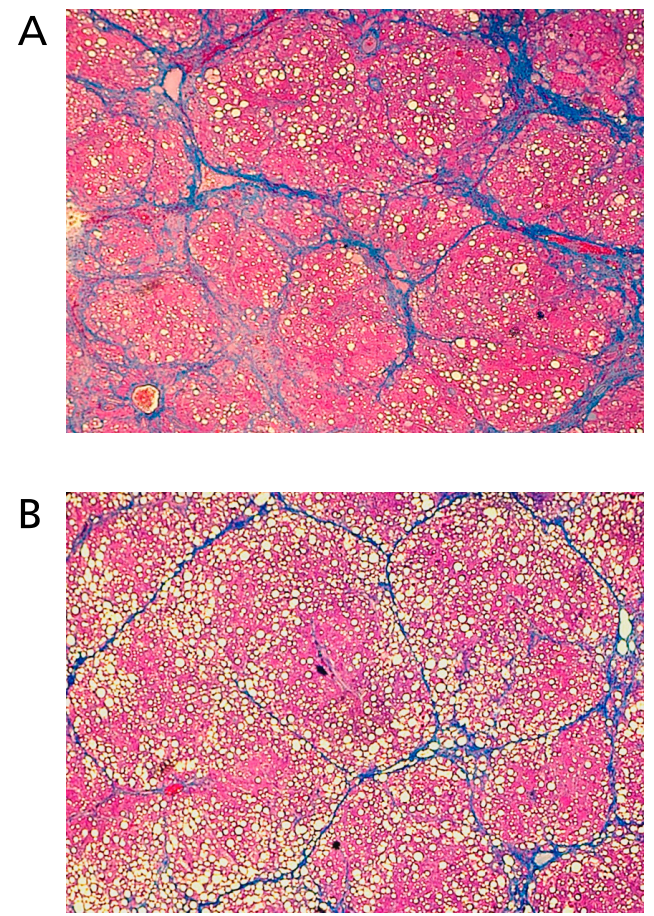

Fig. 2. Effect of BCAA supplementation on liver fibrosis in $\mathrm{CCl}_{4}$-treated rats. $\mathrm{CCl}_{4}$-treated rats were fed either the control diet (control group) or the BCAA-supplemented diet (BCAA group) for 11 weeks. Representative Azan staining sections from liver of rats in control $(A)$ and $B C A A(B)$ groups are shown (Original magnification $\times 40$ ).

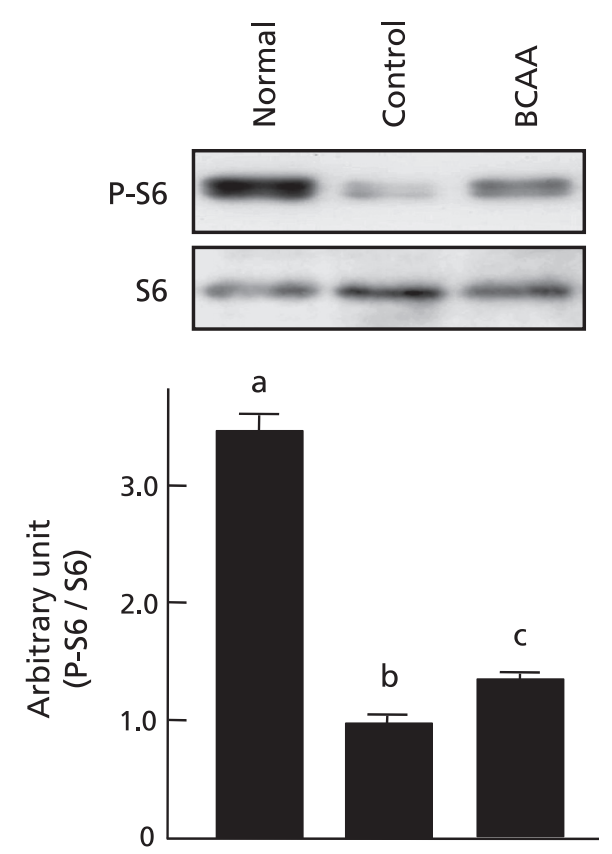

Fig. 3. Effect of BCAA supplementation on phosphorylation of ribosomal protein $\mathrm{S} 6$ in livers of $\mathrm{CCl}_{4}$-treated rats. $\mathrm{CCl}_{4}$-treated rats were fed either the control diet (control group) or the BCAA-supplemented diet (BCAA group) for 11 weeks. Normal rats were fed the control diet for 11 weeks. The phosphorylation of hepatic ribosomal protein S6 was analyzed by western blot using anti-phospho-S6 (P-S6) and anti-S6 (S6) antibodies. The corresponding densitometric analysis is shown. Values are given as mean \pm SEM (normal, $n=3$; control group, $n=5$; BCAA group, $n=6$ ). Means not sharing the same letter are significantly different from each other, $p<0.05$. 

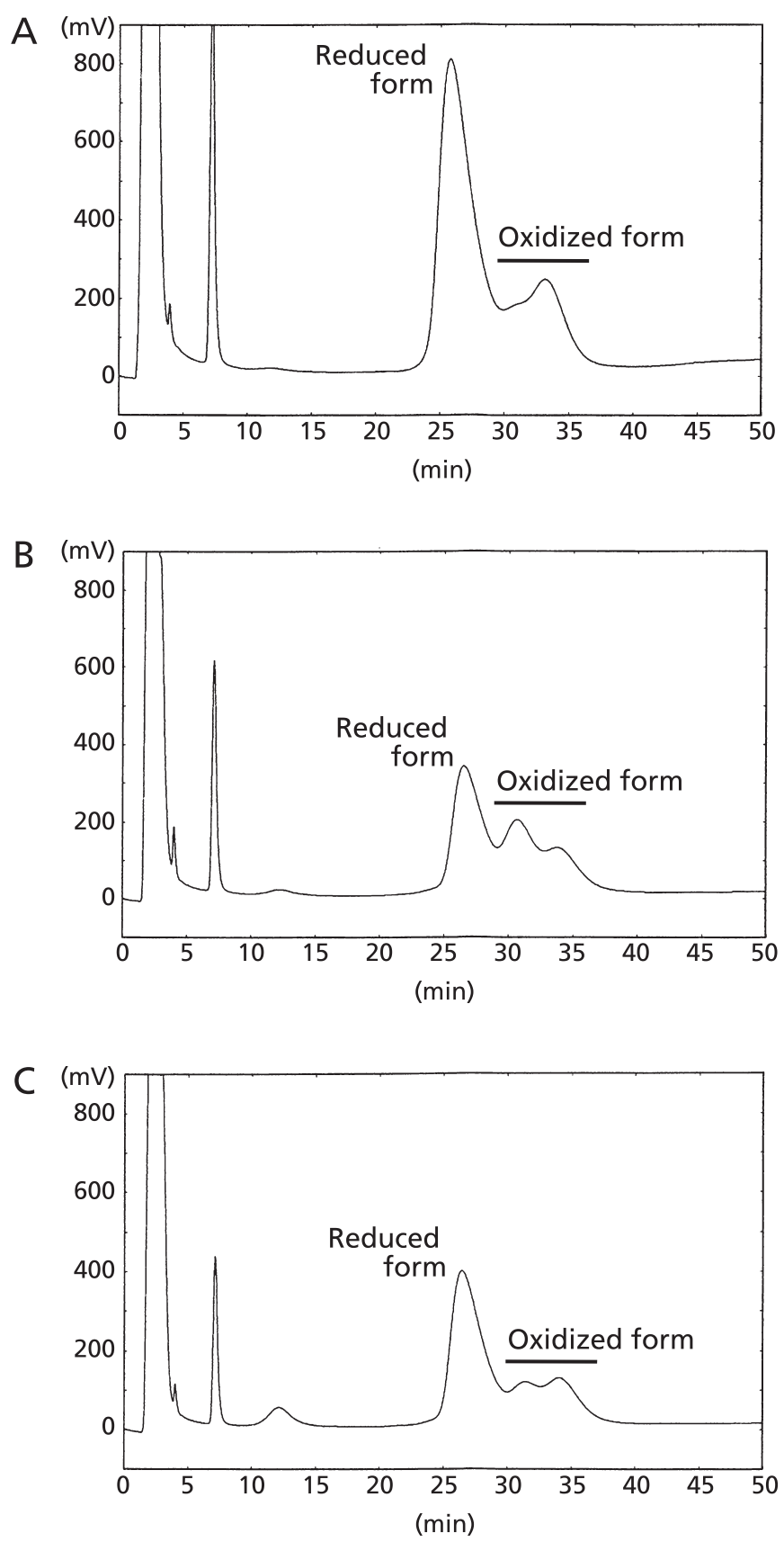

Fig. 4. Typical HPLC profiles of plasma albumin from normal rats (A) and $\mathrm{CCl}_{4}$-treated rats that were fed the control diet (B) or the BCAAsupplemented diet (C) for 11 weeks. HPLC conditions were the same as those in Fig. 1B.

activation of hepatic mTOR signaling. ${ }^{(15,16)}$ Oral administration of BCAA reportedly induces phosphorylation of p70 S6 kinase and 4E-BP1 in livers of both normal ${ }^{(20-22)}$ and cirrhotic rats. ${ }^{(17)}$ When starved rats were administered BCAA, phosphorylation of p70 S6 kinase and 4E-BP1 reached maximum levels within $1 \mathrm{~h}$ and returned to baseline levels within $6 \mathrm{~h}$ after administration. ${ }^{(17,21)}$ All these studies therefore examined the effects of transient administration of BCAAs on hepatic mTOR signaling in food-deprived (16-24 h) rats. ${ }^{(17,20-22)}$ Conversely, our previous study showed that phosphorylation of ribosomal protein S6 could be detected through mTOR signaling in the livers of rats fed freely. ${ }^{(23)}$ To the

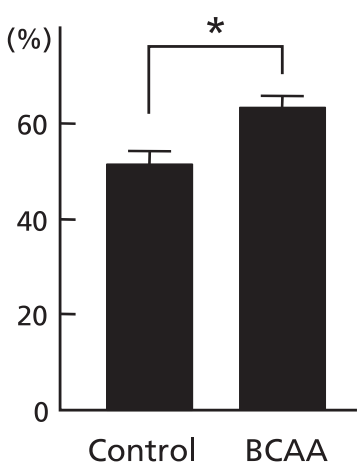

Fig. 5. Effect of BCAA supplementation on percentages of reduced albumin within total albumin in plasma of $\mathrm{CCl}_{4}$-treated rats. $\mathrm{CCl}_{4-}$ treated rats were fed either the control diet (control group) or the BCAA-supplemented diet (BCAA group) for 11 weeks. Plasma albumin was analyzed by HPLC. HPLC conditions were same as those in Fig. 1B. Values are given as means \pm SEM (control group, $n=5$; BCAA group, $n=6) .{ }^{*} p<0.05$.

best of our knowledge, the present results comprise the first reported evidence that continuous supplementation with BCAAs induced phosphorylation of ribosomal protein S6 in the livers of rats with chronic liver disease.

The decrease in the percentage of reduced albumin was attenuated in the plasma of $\mathrm{CCl}_{4}$-treated rats following $\mathrm{BCAA}$ supplementation. A recent study suggested that BCAA supplementation increases the expression of genes involved in antioxidant defense and reduces the production of reactive oxygen species in cardiac and skeletal muscles, but not in the liver of middle-aged mice. ${ }^{(24)}$ In the present study, no significant difference in oxygen radical absorbance capacity in plasma was seen between the control and BCAA groups (data not shown). However, we cannot exclude the possibility that BCAA supplementation increased the percentages of reduced albumin in the plasma of $\mathrm{CCl}_{4}$-treated rats by activating antioxidative mechanisms rather than the albumin synthesis system.

In HPLC analysis of human albumin, three peaks are detected in the order of HMA, HNA1, and HNA2. ${ }^{(8,12)}$ In plasma albumin from healthy subjects, HMA accounts for $70 \%$ and HNA1 for $30 \%$, with HNA2 only barely apparent. In the present study, the second peak that corresponded to HNA1 was small, and the third peak corresponding to HNA2 was large in plasma albumin from normal rats. Future studies need to determine detailed structures of the rat albumin in these peaks. BCAA supplementation in cirrhotic patients significantly increased HMA and significantly decreased HNA1, although HNA2 did not show significant change. ${ }^{(12)}$ The HPLC profiles of the BCAA group in the present study seem to correspond to the result for patients supplemented with BCAAs. The increase in percentages of reduced albumin may thus improve albumin function, including ligand-binding properties and antioxidant activity.(25)

In the present study, the concentration of plasma albumin did not differ significantly between control and BCAA groups, probably because the experimental period was short. However, continuous supplementation with BCAAs for rats with chronic liver disease induced the phosphorylation of ribosomal protein S6 in liver and improved the oxidized/reduced ratio of plasma albumin. Albumin molecules will structurally change the reduced form to the oxidized form for the extended half-life of albumin in cirrhotic patients. ${ }^{(7)}$ Supplementation with BCAAs may improve abnormal albumin metabolism through the activation of albumin synthesis. Actually, analyzing the oxidized/reduced ratio of plasma albumin with every clinical examination in hospital will be difficult. However, if a molecule can be found that reflects the 
oxidized/reduced ratio of plasma albumin in cirrhotic patients, such a molecule might offer a new marker to evaluate the effects of BCAA treatment.

\section{Acknowledgments}

This work was supported in part by a grant to M.K. from the Iijima Memorial Foundation for the Promotion of Food Science and Technology.

\section{References}

1 Fischer JE, Yoshimura N, Aguirre A, et al. Plasma amino acids in patients with hepatic encephalopathy. Effects of amino acid infusions. Am J Surg 1974; 127: 40-47.

2 Rosen HM, Yoshimura N, Hodgman JM, Fischer JE. Plasma amino acid patterns in hepatic encephalopathy of differing etiology. Gastroenterology 1977; 72: 483-487

3 Muto Y, Yoshida T, Yamatoh M. Clinical assessment of nutritional status in patients with liver cirrhosis with special reference to plasma amino acid imbalance. J Clin Biochem Nutr 1986; 1: 89-95.

4 Caregaro L, Alberino F, Amodio P, et al. Malnutrition in alcoholic and virusrelated cirrhosis. Am J Clin Nutr 1996; 63: 602-609.

5 Alberino F, Gatta A, Amodio P, et al. Nutrition and survival in patients with liver cirrhosis. Nutrition 2001; 17: 445-450.

6 Tajika M, Kato M, Mohri H, et al. Prognostic value of energy metabolism in patients with viral liver cirrhosis. Nutrition 2002; 18: 229-234.

7 Moriwaki H, Miwa Y, Tajika M, Kato M, Fukushima H, Shiraki M Branched-chain amino acids as a protein- and energy-source in liver cirrhosis. Biochem Biophys Res Commun 2004; 313: 405-409.

8 Oettl K, Marsche G. Redox state of human serum albumin in terms of cysteine34 in health and disease. Methods Enzymol 2010; 474: 181-195.

9 Watanabe A, Matsuzaki S, Moriwaki H, Suzuki K, Nishiguchi S. Problems in serum albumin measurement and clinical significance of albumin microheterogeneity in cirrhotics. Nutrition 2004; 20: 351-357.

10 Sato S, Watanabe A, Muto Y, et al. Clinical comparison of branched-chain amino acid (L-Leucine, L-Isoleucine, L-Valine) granules and oral nutrition for hepatic insufficiency in patients with decompensated liver cirrhosis (LIV-EN study). Hepatol Res 2005; 31: 232-240.

11 Muto Y, Sato S, Watanabe A, et al. Effects of oral branched-chain amino acid granules on event-free survival in patients with liver cirrhosis. Clin Gastroenterol Hepatol 2005; 3: 705-713.

12 Fukushima H, Miwa Y, Shiraki M, et al. Oral branched-chain amino acid supplementation improves the oxidized/reduced albumin ratio in patients with liver cirrhosis. Hepatol Res 2007; 37: 765-770.

13 Avruch J, Long X, Ortiz-Vega S, Rapley J, Papageorgiou A, Dai N. Amino acid regulation of TOR complex 1. Am J Physiol Endocrinol Metab 2009, 296: E592-E602.

14 Ma XM, Blenis J. Molecular mechanisms of mTOR-mediated translational control. Nat Rev Mol Cell Biol 2009; 10: 307-318.

\section{Abbreviations}

AAA aromatic amino acid

BCAA branched-chain amino acid

$\mathrm{CCl}_{4} \quad$ carbon tetrachloride

HMA human mercaptalbumin

HNA human nonmercaptalbumin

HPLC high-performance liquid chromatography

mTOR mammalian target of rapamycin

15 Ijichi C, Matsumura T, Tsuji T, Eto Y. Branched-chain amino acids promote albumin synthesis in rat primary hepatocytes through the mTOR signal transduction system. Biochem Biophys Res Commun 2003; 303: 59-64.

16 Kuwahata M, Yoshimura T, Sawai Y, et al. Localization of polypyrimidinetract-binding protein is involved in the regulation of albumin synthesis by branched-chain amino acids in HepG2 cells. J Nutr Biochem 2008; 19: 438447.

17 Matsumura T, Morinaga Y, Fujitani S, Takehana K, Nishitani S, Sonaka I. Oral administration of branched-chain amino acids activates the mTOR signal in cirrhotic rat liver. Hepatol Res $2005 ; 33$ : 27-32.

18 Sarwar G, Botting HG. Rapid analysis of nutritionally important free amino acids in serum and organs (liver, brain, and heart) by liquid chromatography of precolumn phenylisothiocyanate derivatives. J Assoc Off Anal Chem 1990; 73: $470-475$.

19 Hayashi T, Suda K, Imai H, Era S. Simple and sensitive high-performance liquid chromatographic method for the investigation of dynamic changes in the redox state of rat serum albumin. J Chromatogr B Analyt Technol Biomed Life Sci 2002; 772: 139-146.

20 Anthony TG, Anthony JC, Yoshizawa F, Kimball SR, Jefferson LS. Oral administration of leucine stimulates ribosomal protein mRNA translation but not global rates of protein synthesis in the liver of rats. $J$ Nutr 2001; 131: 1171-1176.

21 Yoshizawa F, Sekizawa H, Hirayama S, Hatakeyama A, Nagasawa T, Sugahara K. Time course of leucine-induced 4E-BP1 and S6K1 phosphorylation in the liver and skeletal muscle of rats. J Nutr Sci Vitaminol (Tokyo) 2001; 47: $311-315$.

22 Reiter AK, Anthony TG, Anthony JC, Jefferson LS, Kimball SR. The mTOR signaling pathway mediates control of ribosomal protein mRNA translation in rat liver. Int J Biochem Cell Biol 2004; 36: 2169-2179.

23 Kuwahata M, Kuramoto Y, Sawai Y, et al. Polypyrimidine tract-binding protein is involved in regulation of albumin synthesis in response to food intake. J Nutr Sci Vitaminol (Tokyo) 2008; 54: 142-147.

24 D'Antona G, Ragni M, Cardile A, et al. Branched-chain amino acid supplementation promotes survival and supports cardiac and skeletal muscle mitochondrial biogenesis in middle-aged mice. Cell Metab 2010; 12: 362-372.

25 Kawakami A, Kubota K, Yamada N, et al. Identification and characterization of oxidized human serum albumin. A slight structural change impairs its ligand-binding and antioxidant functions. FEBS J 2006; 273: 3346-3357. 